\title{
Hexagonal arrays for radio interferometers
}

\author{
L. G. Sodin ${ }^{1}$ and L. E. Kopilovich ${ }^{2}$ \\ 1 Institute of Radio Astronomy, Kharkov 61002, Ukraine \\ e-mail: sod@ira.kharkov.ua \\ 2 Institute of Radio Physics and Electronics, Kharkov 61085, Ukraine \\ e-mail: kopil@ire.kharkov.ua
}

Received 11 April 2002 / Accepted 4 June 2002

\begin{abstract}
A method for arranging the interferometer elements in nodes of a regular hexagonal coordinate net that provides a nonredundant coverage of a spatial-frequency domain of hexagonal shape is considered. The procedure of synthesizing such arrays based on the use of cyclic difference sets is described. The basic characteristics of the synthesized beam (the beamwidth and sidelobe level) are investigated.
\end{abstract}

Key words. instrumentation: interferometers

\section{Introduction}

The problem of synthesizing configurations of multielement interferometers is widely discussed in connection with the elaboration of new generations of radio telescopes with a high sensibulity and resolution. In a series of papers (Keto 1997; Lonsdale \& Cappallo 1999; Kogan 2000; Boone 2001, 2002), various approaches to searching for optimal antenna arrangement are suggested.

When synthesizing an interferometer configuration, the choice of the optimal variant is based on three requirements:

1. Ensuring the given resolution that determines the cross sizes of the region of the antenna arrangement;

2. minimization of the sidelobe level (SLL) of the synthesized beam in the field of view (FOV) of the interferometer;

3. maximization of the FOV ensured by a correct choice of the minimum distance between the neighbouring antennae.

To implement the second requirement, two approaches are used to estimate the quality and to find the best variant:

2a. The calculation and minimization of the SLL;

$2 b$. the control of positions of the "holes" in the spatialfrequency $(u, v)$ domain. In this case, the well-known rule is used: one should fill densely the central part of the $u, v$ domain, with a decrease in filling towards its periphery; however, avoiding the creation of big "holes".

The third requirement can be best met by placing the antennae in the nodes of a hexagonal grid. At this point, given the minimum distance, $d$, between antennae, the equivalent distance determining the zone free from large grate lobes (GLs) does not exceed the quantity $d \sqrt{3} / 2$ (further, it is usually assumed that $d=1$ ). Besides, the number of GLs in the whole

Send offprint requests to: L. Sodin, e-mail: sod@ira.kharkov.ua

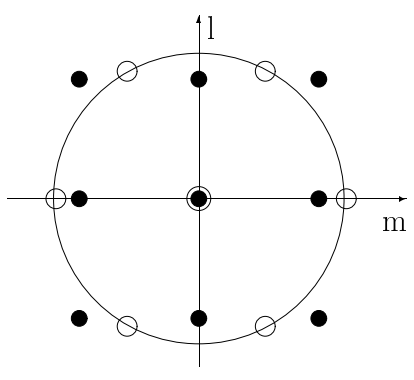

Fig. 1. The beam arrangement for the antenna placing on a square grid (the dark circles) and hexagonal grid (the light circles). The main beam is in the centre.

space is less than for a rectangular grid. The arrangement of the main beam and GLs in space for both types of antenna location is shown in Fig. 1. One can see that the "hexagonal" arrangement provides a somewhat larger FOV.

Also note that when arranging antennae in a regular way, one eliminates the appearance of close baselines giving little information, and simplifies building the system as well as calculating its characteristics. To this end, the hexagonal configuration provides a high circular symmetry of the interferometer beam.

\section{The types of arrays on hexagonal grids}

In the papers of Sodin \& Kopilovich $(2000,2001)$ the variants of the interferometer constructions that do not use the Earth rotation (snapshot regime) and enable one to synthesize arrays of any size by a unified approach are considered. In such a construction the element configuration on a hexagonal grid of radius $r$ provides complete coverage of the hexagonal domain of 
Table 1. The maximum values of the number of elements, $n$, of nonredundant arrays that can be arranged on hexagons of radii $r ; N$ and $N_{\text {tot }}$ are defined by Eqs. (3) and (4).

\begin{tabular}{cccccc}
\hline \hline$r$ & 7 & 12 & 22 & 51 & 83 \\
$n$ & 18 & 28 & 48 & 96 & 150 \\
$N_{\text {tot }}$ & 315 & 900 & 2970 & 15759 & 42273 \\
$N$ & 153 & 378 & 1128 & 4560 & 11175 \\
\hline
\end{tabular}

double radius $R=2 r$ in the $u, v$-plane. The suggested arrays are regular, simply constructed, and have an enhanced FOV. The beamshape of the synthesized interferometer and its sidelobes (SLs) are easily calculated and can be controlled by weighting the correlation products (Sodin \& Kopilovich 2000). One can minimise the number of antennae, given the resolution, if using the hexagonal configurations suggested by Kopilovich (2001). Such configurations can be simply built for any number of elements, and the total length of the communications joining the elements is comparatively small.

The drawback of all these arrays is their redundancy: many baselines are repeated which leads to the need to increase the number of antennae. If the required number of spacings is $N$, then to obtain them, $n$ antennae are required, where

$n=\sqrt{2 N / \alpha}$

$\alpha \approx 0.5$ (Sodin \& Kopilovich 2000, 2001).

One can avoid this drawback when using nonredundant hexagonal structures (in this case $\alpha=1$ ).

As numerous estimates show, on a hexagonal grid of radius $r$ a nonredundant $n$-element array can be arranged, where $n \simeq 2 r$.

For $n<50$, the factor in Eq. (2) is somewhat larger than 2 while for large $n$ it decreases.

The number of obtained baselines is

$N=n(n-1) / 2 \simeq 2 r^{2}$,

that is nearly one-third of the total number of different baselines among the nodes of the grid, $N_{\text {tot }}$ :

$N_{\text {tot }}=3 R(R+1) / 2=3 r(2 r+1) \simeq 6 r^{2}$.

While synthesizing, one should strive to maximize $n$, given the value of $r$. The maximum obtained values of $n$ are given in Table 1.

\section{Synthesis of nonredundant arrays on hexagonal grids}

The synthesis procedure must give a maximum-element array on a grid of given radius $r$ (that is equivalent to arranging given number of elements on a grid of the minimum radius). The method of random searching for such arrays on hexagons (Golay 1971) is effective only for grids of a small size. Here, we employ the method based on using cyclic difference sets (CDSs) (Kopilovich 1998a, 1998b).

A CDS with the parameters $V, k, \Lambda$ is a set $\left\{d_{j}\right\}$ of $k$ integers, $0 \leq d_{1}<d_{2}<\ldots<d_{k}<V$, such that any positive integer $\mu<V$ possesses exactly $\Lambda$ representations of the form $d_{i}-d_{j} \bmod V$ (Baumert 1971). Here, we will make use of these sets with $\Lambda=1$.

A CDS with $\Lambda=1$ represents a nonredundant array on a linear segment $\left[0, d_{k}\right]$; at this point, $V=k^{2}-k+1$. Such CDSs exist for any $k=q+1$, where $q$ is a prime or a power of a prime, i.e. for

$k=3,4,5,6,8,9,10,12,14,17,18, \ldots$.

In what follows, it is important that for each $k$ there exist many so-called equivalent sets that can be obtained from some initial one by multiplying it by $t$ coprime with $V,((t, V)=1)$, and by shifting it by $s$ (the operations are carried out modulo $V$ ):

$d_{i}^{\prime} \equiv\left(d_{i} \cdot t+s\right) \bmod V$.

All these sets are CDSs with the same parameters. This enables one to select among them a set giving the best solution to the problem. One can take as initial any of the equivalent sets that allows one to make use of the CDS tables (these are available from the monographs of Baumert 1971 and Kopilovich \& Sodin 2001).

We now describe the synthesis procedure briefly. It consists of three stages.

1. The folding of some initial CDS, with $\Lambda=1$ and given $k$, onto a square grid of size $B \times B$, where $B$ must satisfy the conditions

$B \geq 2 r+1, B^{2}>d_{k}+1$.

To this end, the coordinates of the set elements on the square grid are

$x_{i} \equiv d_{i} \bmod B, y_{i}=\operatorname{Entier}\left(d_{i} / B\right)$.

2. The calculation of the number of the elements $\left(x_{i}, y_{i}\right)$ within the region

$|x-y| \leq r$

of the inner square of size $(2 r+1) \times(2 r+1)$.

3. The transformation of the obtained set in the region (9) to the set on a hexagonal grid by the formulas

$x_{h i}=x_{i}-0.5\left(y_{i}+r\right), y_{h i}=\sqrt{3}\left(y_{i}-r\right) / 2$.

Folding consecutively all sets of the CDS ensemble on squares with various sidelengths $B$ meeting the conditions (7), one finds the maximum-element nonredundant set in the region (9), and hence in a hexagon of radius $r$.

We now show an example of the construction for $r=7$, $n=18$. The CDS with the parameters $V=307, k=18, \Lambda=1$ :

$\left\{d_{i}\right\}=\{0,2,10,22,53,56,82,83,89,98,130,148$,

$$
153,167,188,192,205,216\}
$$

from the table in Kopilovich \& Sodin (2001) was taken as the initial set, and several 18-element arrays on the hexagonal grid of radius $r=7$ were obtained. To obtain one of them we first transformed the set (11) by Eq. (6) with $t=59, s=92$ :

$$
\left\{d_{i}^{\prime}\right\}=\{18,19,41,61,68,77,87,92,121,124,132,
$$

$149,162,210,214,216,249\}$, 


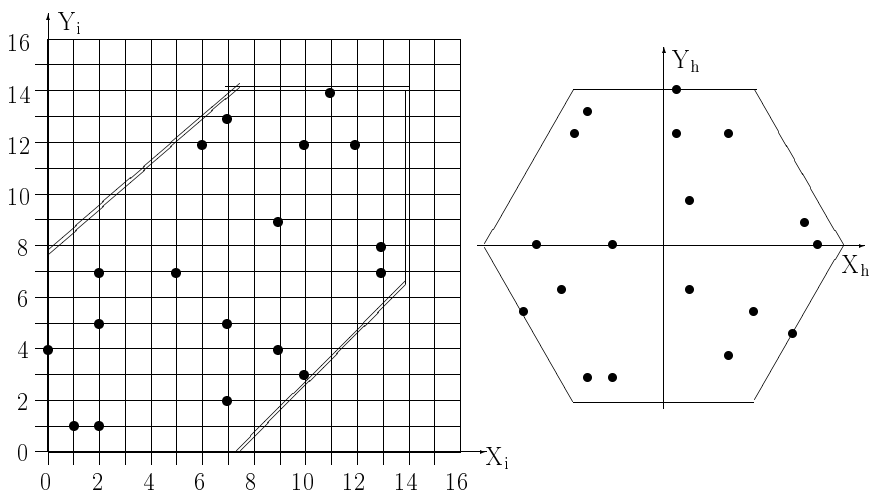

Fig. 2. Left: The element arrangement after folding the CDS (11) onto the square with sidelength $B=17$; here, $n=18$, and the region $|x-y| \leq 7$ is outlined. Right: The element arrangement on the hexagon of radius $r=7$ obtained by the transformation (10) of the outlined region.

and then folded the set (12) onto the square with sidelength $B=17$. The obtained array on the square grid is shown in Fig. 2 (left), and the transformed one on the hexagonal grid is shown in Fig. 2 (right). In this example, one succeeded in transferring all the CDS elements to a hexagon of the given radius; here $n=2 r+4$, that is somewhat better than that which Eq. (2) yields. The array obtained by the suggested method for $r=12, n=28$, and also the baselines in the hexagonal $u, v$ domain of radius $R=24$ are shown in Fig. 3. One can see that the central part of the domain is densely covered while the density decreases towards the periphery. Note that in the described procedure only the simplest arithmetical operations are used.

\section{The width of synthesized beam and the sidelobe level}

In this section, two basic parameters of an interferometer of the suggested type - the width of the synthesized beam and its SLL - are considered. The third important parameter - the field of view, i.e. the domain of the spatial angles free of GLs has been shown in Fig. 1.

The shape of the interferometer beam is defined by the expression

$F(l, m)=\frac{1}{n(n-1)} \sum_{p, q=1,}^{n} \sum_{p \neq q}^{n} \exp \left[\mathrm{i} \frac{2 \pi}{\lambda}\left(u_{p q} l+v_{p q} m\right)\right]$,

where $u_{p q}=x_{p}-x_{q}, v_{p q}=y_{p}-y_{q}, l=\sin \theta \sin \phi, m=$ $\sin \theta \cos \phi, \theta$ is the angle with the normal to the interferometer plane, $\phi$ is the azymuth angle, and $\lambda$ is the wavelength.

When considering the beamshape, it is convenient to pass from the double sum in Eq. (13) to the single one, as supposed by Kogan (1999):

$F(l, m)=\frac{n}{n-1}\left|\frac{1}{n} \sum_{p=1}^{n} \exp \left[\mathrm{i} \frac{2 \pi}{\lambda}\left(x_{p} l+y_{p} m\right)\right]\right|^{2}-\frac{1}{n-1}$.

The first term in Eq. (14) is positive, therefore negative SLs do not exceed $1 /(n-1)$ in absolute value. Hence, maximum SLs

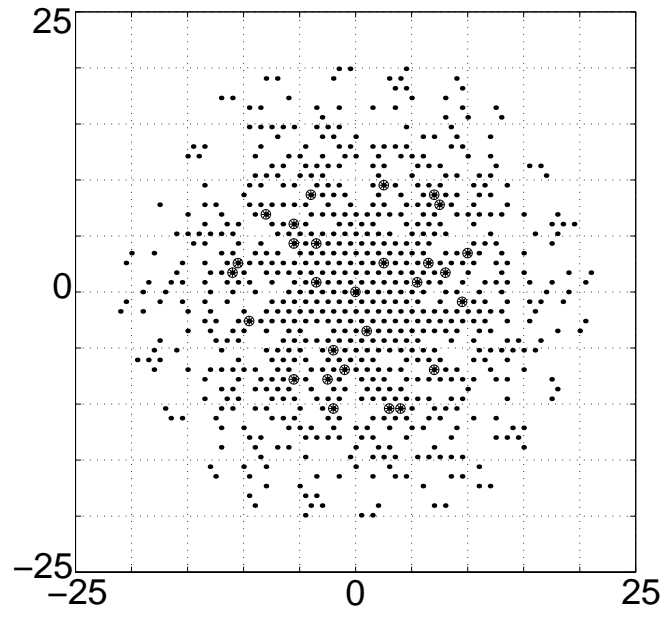

Fig. 3. The arrangement of 28 antennas on the hexagon of radius $r=12$ (dark circles), and the totality of the baselines in the hexagonal domain of radius $R=24$ in the $u, v$-plane (dots).

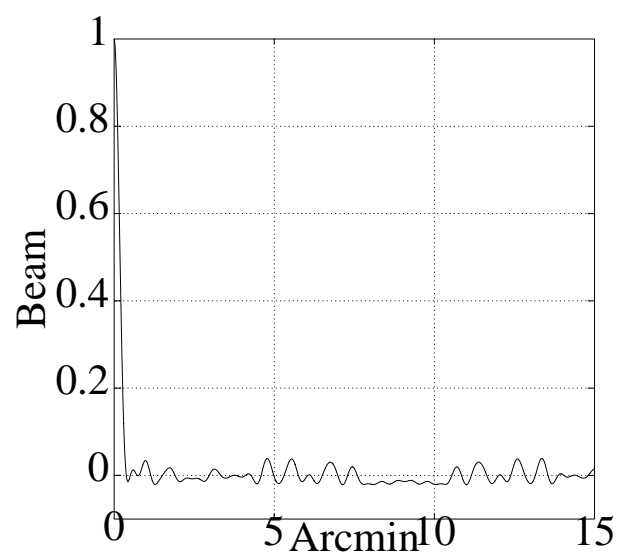

Fig. 4. The beam cross-section $F(0, m)$ for the hexagonal interferometer with $r=22, n=48$.

can be considered as positive bursts of $F(l, m)$ over the zero level. The cross-section $F(0, m)$ of the beam for $r=22, n=48$ is shown in Fig. 4.

To estimate the beamwidth and the SLL, the power patterns for a series of interferometers with various numbers of elements and $d / \lambda=200$ were calculated. The beamwidths of these systems are given in Table 2 and can be described rather well by

$\theta / \operatorname{arcmin} / \simeq 3.6 \times 10^{3} \frac{\lambda}{n d}$

The level of the maximum positive SL is obtained by calculating $F(l, m)$ in a thick net of points $(l, m)$. This procedure is rather long for large $n$, since large SLs are formed in the directions not predictable a priori. The results of the calculation are given in Table 2. One can see that the maximum SLL is rather small and satisfies the requirements of the dynamic range of radio interferometric observations. To this end, almost all SLs are less than the maximum one and close to $1 / n$.

In real conditions, the need for additional restrictions on the region of the antenna location may appear. For example, consider the case when the inner region of the hexagon is forbidden 
Table 2. The beamwidth, $\theta$, and the sidelobe level, $F_{s l}$, of the $n$-element nonredundant interferometers.

\begin{tabular}{ccccccc}
\hline \hline$r$ & 7 & 12 & 22 & 51 & 64 & 83 \\
$n$ & 18 & 28 & 48 & 96 & 120 & 150 \\
$\theta / \operatorname{arcmin} /$ & 1.15 & 0.7 & 0.4 & 0.19 & 0.16 & 0.13 \\
$F_{s l}$ & 0.106 & 0.089 & 0.066 & 0.043 & 0.039 & 0.034 \\
\hline
\end{tabular}

Table 3. The characteristics $\left(\theta, F_{s l}\right)$ of the nonredundant interferometers with the elements arranged in hexagonal rings, with the inner radii $\left(r_{\text {in }}\right)$ equal to nearly half the outer ones $(r)$.

\begin{tabular}{cccccc}
\hline \hline$r$ & 10 & 18 & 27 & 64 & 80 \\
$r_{\text {in }}$ & 6 & 10 & 14 & 33 & 41 \\
$n$ & 24 & 36 & 48 & 96 & 120 \\
$\theta / \operatorname{arcmin} /$ & 0.80 & 0.44 & 0.30 & 0.14 & 0.11 \\
$F_{s l}$ & 0.115 & 0.098 & 0.091 & 0.064 & 0.058 \\
\hline
\end{tabular}

for the antennas. Specifically, the arrangement only within a "hexagonal ring" of the outer radius $r$ and inner radius $r_{\text {in }} \simeq r / 2$ is permitted. At this point, of course, the number of elements is less than for a full hexagon of radius $r$. For this case, the data on the beamwidth and SLL are given in Table 3.

The comparison of the data of Tables 2 and 3 shows that the beamwidth for a hexagonal ring is described rather well by Eq. (15) while the SLL for the ring is higher than for a full hexagon of the same radius.

\section{Conclusions}

1. Nonredundant array configurations on hexagonal grids are suggested. The advantage of such configurations, when using them in interferometers, consists of - a high resolution, given the number of elements;
- a low sidelobe level;

- an enhanced field of view free of large grate lobes.

2. The method for constructing array configurations based on using cyclic diference sets is described.

We think that the suggested structures may be useful for building radio interferometers of various wavelengths.

Acknowledgements. This work was partly supported by the INTAS grant number 97-1964.

\section{References}

Baumert, L. D. 1971, Cyclic Difference Sets. Lect. Notes in Math., vol. 182 (Berlin, Springer)

Boone, F. 2001, A\&A, 377, 368

Boone, F. 2002, in ALMA memo No. 400

Golay, M. E. 1971, JOSA, 61(2), 272

Keto, E. 1997, ApJ, 475, 843

Kogan, L. 1999, PASP, 11, 510

Kogan, L. 2000, IEEE Trans. Antennas and Propag., 48(7), 1075

Kopilovich, L. E. 1998a, J. Mod. Opt., 45(11), 2417

Kopilovich, L. E. 1998b, Kinematics \& Physics of Celestial Bodies, 15(4), 286

Kopilovich, L. E. 2001, A\&A, 380, 758

Kopilovich, L. E., \& Sodin, L. G. 2001, Multielement System Design in Astronomy and Radio Science (Dordrecht, Kluwer)

Lonsdale, C. J., \& Cappallo, R. J. 1999, in Perspectives on Radio Astronomy: Technologies for Large Antenna Arrays, ed. A. B. Smolders, \& M. P. van Haarlem (Netherlands Foundation for Research in Astronomy), 243

Sodin, L. G., \& Kopilovich, L. E. 2000, Radiophys. and Quant. Electron., 43(4), 345

Sodin, L. G., \& Kopilovich, L. E. 2001, A\&A, 368, 747 